\title{
Coding of Shape and Position in Macaque Lateral Intraparietal Area
}

\author{
Peter Janssen, Siddharth Srivastava, Sien Ombelet, and Guy A. Orban \\ Laboratorium voor Neuro- en Psychofysiologie, Katholieke Universiteit Leuven Medical School, B-3000 Leuven, Belgium
}

\begin{abstract}
The analysis of object shape is critical for both object recognition and grasping. Areas in the intraparietal sulcus of the rhesus monkey are important for the visuomotor transformations underlying actions directed toward objects. The lateral intraparietal (LIP) area has strong anatomical connections with the anterior intraparietal area, which is known to control the shaping of the hand during grasping, and LIP neurons can respond selectively to simple two-dimensional shapes. Here we investigate the shape representation in area LIP of awake rhesus monkeys. Specifically, we determined to what extent LIP neurons are tuned to shape dimensions known to be relevant for grasping and assessed the invariance of their shape preferences with regard to changes in stimulus size and position in the receptive field. Most LIP neurons proved to be significantly tuned to multiple shape dimensions. The population of LIP neurons that were tested showed barely significant size invariance. Position invariance was present in a minority of the neurons tested. Many LIP neurons displayed spurious shape selectivity arising from accidental interactions between the stimulus and the receptive field. We observed pronounced differences in the receptive field profiles determined by presenting two different shapes. Almost all LIP neurons showed spatially selective saccadic activity, but the receptive field for saccades did not always correspond to the receptive field as determined using shapes. Our results demonstrate that a subpopulation of LIP neurons encodes stimulus shape. Furthermore, the shape representation in the dorsal visual stream appears to differ radically from the known representation of shape in the ventral visual stream.
\end{abstract}

Key words: shape; extrastriate; macaque; LIP; parietal; saccade

\section{Introduction}

The primate visual system accomplishes its many tasks critical for survival through two routes originating in primary visual cortex: a ventral stream subserving object recognition and a dorsal stream for the analysis of space and planning of actions (Ungerleider and Mishkin, 1982; Goodale and Milner, 1992). Numerous studies have shown that ventral stream areas are specialized in the detection and discrimination of object features such as shape, color, and texture (Logothetis and Sheinberg, 1996; Tanaka, 1996). The shape preference of neurons in the inferotemporal cortex (IT), but not their absolute response level, is typically preserved over stimulus transformations: position, size (Schwartz et al., 1983; Ito et al., 1995), visual cue (Sáry et al., 1993), and occlusion (Kovacs et al., 1995). Hence, IT encodes an abstract and robust representation of shape, essentially unaffected by irrelevant and continuously fluctuating visual information impinging on the retina.

Much less is known about the shape representation in the

Received Feb. 4, 2008; revised May 5, 2008; accepted May 7, 2008.

This work was supported by Neurobotics (European Union Project IST-001917), Fonds voor Wetenschappelijk Onderzoek Grant G.0495.05N, Geneeskundige Stichting Koningin Elisabeth, G0A 2005/18, Human Frontier Science Program Career Development Award Grant 0057/2005-C, and Excellentie Financiering Grant 05/014. We thank Gerrit Meulemans, Piet Kayenbergh, Marc Depaep, Stijn Verstraeten, and Inez Puttemans for technical assistance and Kirsten Vanderheyden for help with the training and single-cell recordings.

Correspondence should be addressed to Peter Janssen, Laboratorium voor Neuro- en Psychofysiologie, Katholieke Universiteit Leuven Medical School, Herestraat 49, bus 1021, B-3000 Leuven, Belgium. E-mail: peter.janssen@med.kuleuven.be.

D01:10.1523/JNEUROSCI.0499-08.2008

Copyright $\odot 2008$ Society for Neuroscience $\quad 0270-6474 / 08 / 286679-12 \$ 15.00 / 0$ dorsal stream. Goodale et al. (1991) described a patient, DF, with visual form agnosia after sustaining a ventral stream lesion. Despite her inability to recognize objects and faces, even to describe the orientation of elongated bars, she was able to grasp objects or slide a card into an open slot at various orientations. Her dorsal visual stream was able to compute the orientation of the slot or the shape of the object (James et al., 2003) and adjusted her hand accordingly.

Anterior intraparietal (AIP) neurons respond selectively to real-world objects (Sakata et al., 1995; Murata et al., 2000), and a large proportion of lateral intraparietal (LIP) neurons shows shape-selective responses (Sereno and Maunsell, 1998; Sereno and Amador, 2006). Lehky and Sereno (2007) reported a greater degree of shape selectivity in IT than in LIP. No previous studies, however, have systematically explored the invariance of the shape preference for changes in position and size, properties well documented in the ventral visual stream and considered a hallmark of genuine shape selectivity.

Previous studies did not investigate the relationship between saccadic activity and shape selectivity. LIP activity has been linked to cognitive control processes such as saccade planning (Snyder et al., 1997), spatial attention (Gottlieb et al., 1998), decision formation (Shadlen and Newsome, 1996), reward expectation (Platt and Glimcher, 1999), and timing (Leon and Shadlen, 2003; Janssen and Shadlen, 2005). The neural population in LIP, however, is by no means homogeneous in terms of spatially selective saccadic and delay period activity. Hence, shape-selective LIP neurons may represent a special class of neurons. Alternatively, 
the reported shape selectivity in LIP might be a byproduct of its salience map of objects that are potential saccade targets. In this view, LIP neurons do not compute an abstract representation of shape as in IT but merely signal where to direct the eyes if salient objects appear in the visual field.

We observed shape tuning in the majority of LIP neurons, but position-invariant shape selectivity was present only in a minority and size invariance was generally weak. Most neurons showed both spatially selective saccadic activity and shape tuning.

\section{Materials and Methods}

\section{Subjects and surgery}

Two male rhesus monkeys (monkey D, $6 \mathrm{~kg}$; monkey E, $6.5 \mathrm{~kg}$ ) were trained to sit in a primate chair. All procedures were performed in accordance with the National Institutes of Health Guide for the Care and Use of Laboratory Animals and were approved by the Ethical Committee at the Katholieke Universiteit Leuven Medical School. Under isoflurane anesthesia and sterile conditions, a head post (Crist Instruments) was implanted on the skull using ceramic screws and dental acrylic. At least 6 weeks after surgery, the monkeys began training in passive fixation and eye movement tasks. After 2-3 months of training, a craniotomy was made, and a magnetic resonance imaging (MRI)compatible recording cylinder was implanted vertically at Horsley-Clark coordinates 5P, 15L over the left hemisphere of monkey D and the right hemisphere of monkey E. To verify the recording positions, we inserted glass capillaries filled with a $2 \%$ copper sulfate solution into the recording grid at several grid positions and acquired structural MR images $(0.5 \mathrm{~mm}$ resolution) (Fig. $1 \mathrm{~A}$ ). These images confirmed that the recording cylinder was implanted over the anterior part of area LIP and indicated that the recording area extended from Horsley-Clark coordinates $3 \mathrm{P}$ to $7 \mathrm{P}$ and from $9 \mathrm{~L}$ to $15 \mathrm{~L}$, covering the entire lateral bank but not the fundus of the intraparietal sulcus (IPS) (Fig. 1B). A second cylinder was implanted over a craniotomy orthogonal to the recording cylinder in the coronal plane of the same hemisphere. This cylinder allowed real-time imaging of the IPS and of the electrode tip during the recordings by means of high-resolution ultrasound (Philips HDI 5000 SonoCT; scan frequency, $17 \mathrm{MHz}$; resolution, $0.5 \mathrm{~mm}$ ) (Fig. $1 \mathrm{~B}$ ). All recordings were verified to be in the lateral bank of the IPS. Finally, we recorded multiunit activity at two recording positions using stainless steel electrodes (1 M $\Omega$; FHC) and made three consecutive electrolytic lesions spaced $1 \mathrm{~mm}$ apart in the lateral bank and the underlying white matter in monkey E. Metal particles were deposited in the brain and were visualized in the MRI, confirming that the recordings were made in the anterior part of LIP.

\section{Stimuli and tests}

Shape-tuning test. The stimuli were a set of 56 simple two-dimensional (2D) shapes varying along dimensions known to be important for grasping (Jenmalm and Johansson, 1997; Goodwin et al., 1998) (Fig. 1C). Five dimensions were implemented as variations imposed on a square: curvature along the vertical boundaries, curvature along the horizontal boundaries, horizontal taper, vertical taper, and aspect ratio. The two additional dimensions consisted of orientation and center-of-mass. Each shape dimension contained two extreme values (e.g., positive and nega-

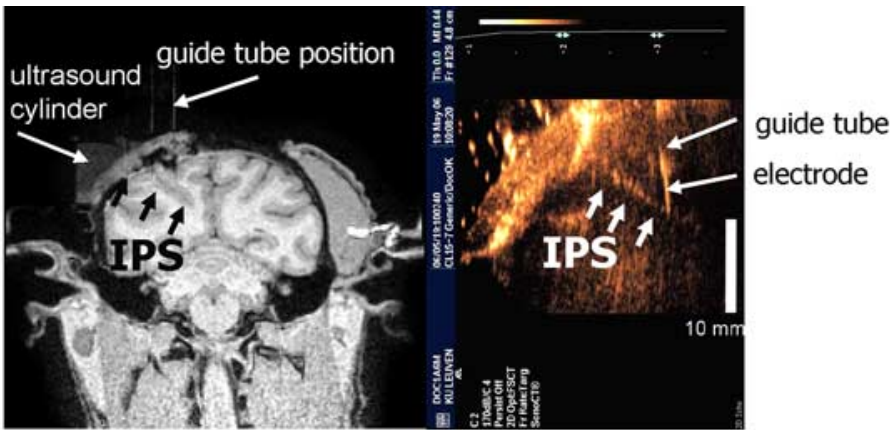

guide tube electrode
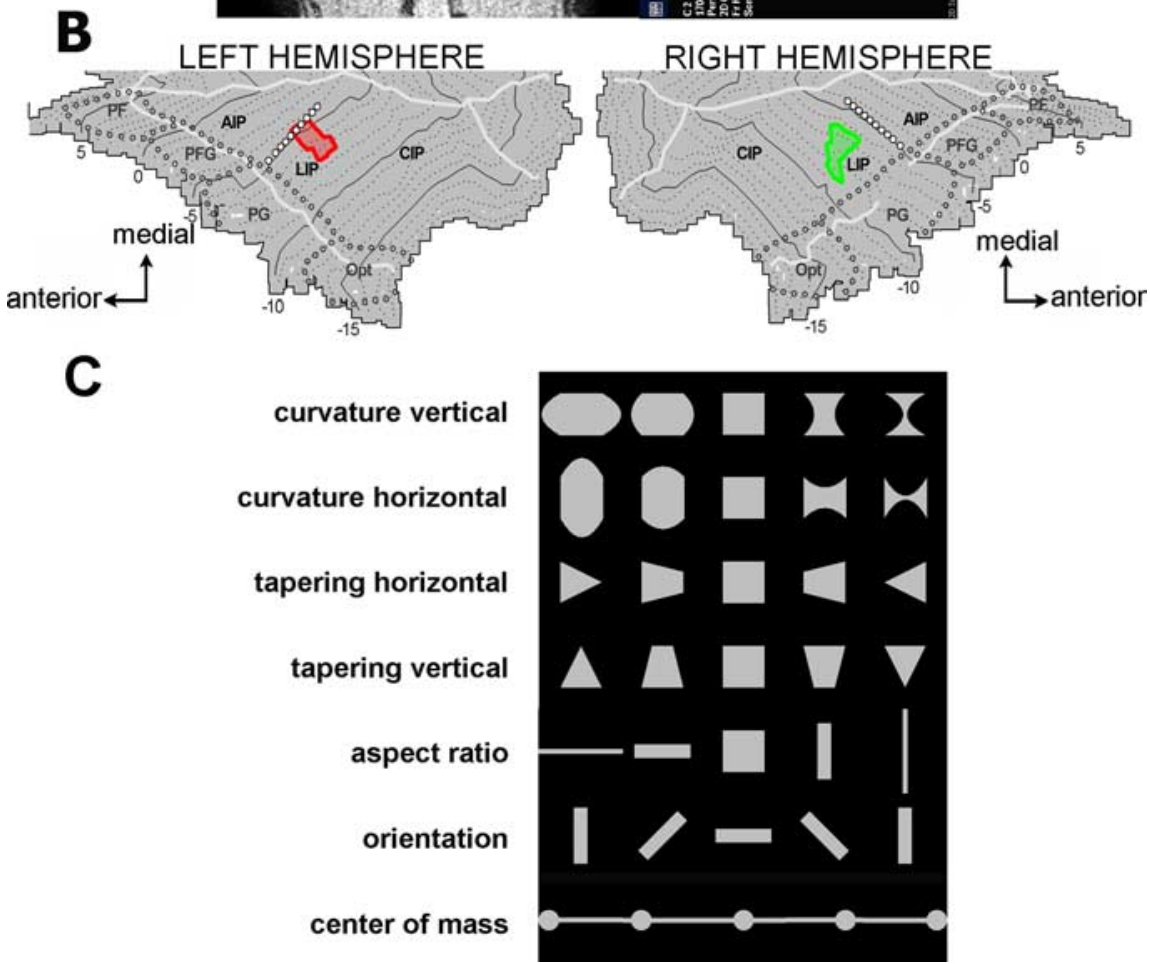

Figure 1. Recording area and stimuli. $A$, Structural MR (left) and ultrasound image (right) of the recording area. The guide tube positions indicated represent two recording positions. $\boldsymbol{B}$, Recording positions projected onto the flat map of the IPS (Durand et al., 2007) for monkey $D$ (left) and monkey $E$ (right). The boundary line denotes the anterior boundary of the activation by visual saccades in an awake monkey functional MRl experiment. C, Stimulus set presented per dimension. Twenty-eight shapes and two different sizes $\left(1\right.$ and $\left.5^{\circ}\right)$ were used.

tive curvature), two intermediate values, and the "neutral" value. The shapes were white (luminance, $34 \mathrm{~cd} / \mathrm{m}^{2}$ ) and were presented on a dark background $\left(0.4 \mathrm{~cd} / \mathrm{m}^{2}\right)$ on a Philips Brilliance 202P4 monitor operating at $120 \mathrm{~Hz}$ at a viewing distance of $86 \mathrm{~cm}$. The stimulus set comprised two sets of 28 shapes: the small shape set averaged $1^{\circ}$ in size (vertical and horizontal diameter range, $0.25-4^{\circ}$ ), whereas the large shapes averaged $5^{\circ}$ (vertical and horizontal diameter range, $0.65-10^{\circ}$ ). We did not attempt to match surface area within the stimulus sets, but each shape was matched with at least one other shape with identical surface area but rotated between 45 and $180^{\circ}$.

In the shape-tuning test, the monkey performed a passive fixation task. A trial started with the onset of a small fixation target in the center of the display. If the animal's eye position remained within $1.5^{\circ}$ of the fixation point for $400 \mathrm{~ms}$, a shape was presented for $500 \mathrm{~ms}$. If the animal continued to fixate for another $100 \mathrm{~ms}$ after stimulus offset, a drop of water was delivered as a reward. The monkeys were never allowed to make eye movements toward the shapes.

Shape-position test. In the shape-position test, two shapes were presented at 35 positions, spaced $2^{\circ}$ apart, in the contralateral hemifield. The shapes were selected from the entire shape set based on the neural responses in the shape-tuning test: one shape was selected that elicited a strong response from the neuron, whereas the second evoked only weak 
responses. A subset of the neurons was tested with two shapes presented at the fovea and at 10 positions (at $0,45,90,135$, and $180^{\circ}$ ) on the perimeter of two circles with radii of 4.5 and $9^{\circ}$. The monkeys' task was to fixate a small spot on the display, but, unlike those in the shape-tuning test, these stimuli were presented consecutively for $400 \mathrm{~ms}$ as long as the monkey fixated. The interstimulus interval was $300 \mathrm{~ms}$.

Saccade tests. Three types of delayed saccade tests (Hikosaka and Wurtz, 1983) were used. In the saccade-mapping test, the animal had to fixate a small spot in the center of the display for $400 \mathrm{~ms}$, after which a saccade target $\left(0.25^{\circ}\right.$ white spot) was presented at one of 10 positions in the contralateral hemifield on the perimeters of two circles with radii of 4.5 and $9^{\circ}\left(\right.$ at $0,45,90,135$, and $\left.180^{\circ}\right)$. After a variable delay (between 100 and $2500 \mathrm{~ms}$ ), the fixation point dimmed, indicating to the animal that it should make a saccade to the target. In the target-cue test, both a (green) saccade target and a (blue) go cue of equal luminance $\left(20 \mathrm{~cd} / \mathrm{m}^{2}\right)$ appeared simultaneously after $400 \mathrm{~ms}$ of stable fixation. After a variable delay (between 100 and $2500 \mathrm{~ms}$ ), the dimming of the go cue was the signal to make an eye movement to the saccade target. Target and go cue could appear either inside the receptive field (RF) of the neuron or in the opposite hemifield (Janssen and Shadlen, 2005). Finally, in the memoryguided saccade task, a target was flashed briefly $(50 \mathrm{~ms})$ either within or outside of the RF of the neuron. After a variable delay, the dimming of the fixation point served as the go signal for making an eye movement to the remembered target location.

Recording procedure. The position of the right eye was sampled at 500 $\mathrm{Hz}$ with an EyeLink 1000 eye tracker (SR Research). A photocell was attached to the monitor to detect a white square in the lower left corner of the screen that appeared in the first video frame containing the stimulus (either a shape or a saccade target). We inserted tungsten microelectrodes (impedance at $1 \mathrm{kHz}: 1 \mathrm{M} \Omega$ ) by means of a hydraulic microdrive (FHC) through a stainless steel guide tube. Neural activity was amplified and filtered between 300 and $5000 \mathrm{~Hz}$. Eye position signals, neural activity, and photocell pulses were digitized and processed at $20 \mathrm{kHz}$ on a digital signal processor (C6000 series; Texas Instruments). Spikes were discriminated on-line on the DSP using a dual time-window discriminator and displayed using LabView and custom-designed software. During the recordings, the ultrasound cylinder was filled with sterile conductive gel (Pharmaceutical Innovations), and both the IPS and the microelectrode were visualized by manually holding the ultrasound probe on the cylinder. The trajectory of the electrode as seen on the ultrasound monitor correlated perfectly with the transitions between silent and active zones as judged by listening to the sound of the speakers.

We searched for responsive neurons while the monkey performed either the fixation task or the visually guided saccade task. In a typical recording session, the electrode was lowered until it reached the lateral bank of the IPS while the monkey made visually guided saccades. These initial recordings were based on the transition between active and silent zones as the electrode traversed the white matter, the medial bank, the sulcus, and finally the lateral bank of the IPS. After verifying that the tip of the electrode was positioned in the lateral bank of the IPS with ultrasound imaging, we searched for the RF of the multiunit activity by manually placing either saccade targets or shapes at various locations in the contralateral hemifield until a consistent neural response was observed. Formal testing started once an LIP unit could be well isolated, usually in the order shape-tuning test $\rightarrow$ shape-position test $\rightarrow$ saccade test.

\section{Data analysis}

Shape tuning. Baseline firing rate was calculated from the mean spike rate in the $400 \mathrm{~ms}$ interval before stimulus onset on a trial-by-trial basis. Net neural responses were computed by subtracting the baseline firing rate from the mean activity between 40 and $360 \mathrm{~ms}$ after stimulus onset. We computed separate two-way ANOVAs for the neural responses for each dimension, with factors dimension and size. A neuron was judged to be tuned for 2D shape if, for at least one of the dimensions tested, either the main effect of dimension $(p<0.01)$ or the interaction between dimension and size $(p<0.05)$ were significant. We will reserve the expression "tuned for 2D shape" merely to indicate the presence of significant response differences within a dimension. The term "shape selectivity" will be used to indicate significant response difference based on a coding of shape, i.e., invariant over changes in size and/or position.

The shape-tuning index (STI) was computed as STI = (best - worst)/ best, in which best is the strongest response of the neuron (the preferred shape), and worst is the weakest response of the neuron to another shape along the same dimension and of the same size (the nonpreferred shape). The latency of the neural response was calculated using the response to the preferred shape. For each neuron, the distribution of the number of spikes summed over all trials in each of the $10 \mathrm{~ms}$ bins in the $400 \mathrm{~ms}$ preceding stimulus onset was fitted with a Poisson function (Maunsell and Gibson, 1992; Bisley et al., 2004). We calculated a cutoff from the Poisson fit as the number of spikes below which the background spike count was expected to lie $95 \%$ of the time. The time of the first of two consecutive bins with a spike count higher than or equal to this cutoff value was considered to be the latency of the neuron. For the population analysis, all presentations of the preferred shape from all neurons were grouped, and the same analysis was used using $1 \mathrm{~ms}$ bins. To take the frame rate of the monitor into account ( $8.3 \mathrm{~ms}$ per frame), we added 4.2 $\mathrm{ms}$ to the calculated latency value, i.e., the average time interval between the appearance of the stimulus and the detection of the square at the bottom of the monitor at the end of each frame.

Size invariance. For each neuron that showed a significant response to both sizes in the test ( $t$ test, $p<0.05$ ), we ranked the shapes of the preferred size according to the normalized net response (rank 1 , shape to which the neuron showed the largest response; rank 28, shape to which the neuron showed the weakest response). We then applied the same ranking to the corresponding shapes of the nonpreferred size and computed the average ranked responses across neurons for both sizes separately. If the population of neurons preserves its selectivity for different sizes, the two curves will be parallel. Linear regression analysis was used to estimate the slopes and confidence intervals of the curve relating average neural response to shape rank for the preferred and the nonpreferred sizes.

To quantify the degree of size invariance, we computed the separability of size and shape, analogous to other studies in the ventral visual stream (Brincat and Connor, 2004). Briefly, the index of separability is the correlation between the observed responses and the predicted responses when one assumes perfect separability of the two dimensions studied (in this case, shape and size). For our experiment, the predicted responses were computed on a matrix with two rows (preferred and nonpreferred size) and 28 columns (the shapes in the test). Each entry of the matrix contained the average net response of the neuron to a shape of a given size. The predicted responses are simply the product of the marginal sums of each row and each column. The correlation coefficient between predicted and observed responses represents the index of separability. An index of 1 indicates perfect separability, i.e., invariance of shape selectivity over size changes, whereas an index of 0 indicates that the neural selectivity changes markedly for different sizes.

Position invariance. Contour plots were constructed by twodimensional linear interpolation of the mean normalized responses to preferred and nonpreferred shape in the shape-position test. On the interpolated maps, we calculated the size of the RF (the area above $50 \%$ of the maximal response), the center-of-mass of the RF, and the distance between the center-of-mass of the RF for preferred and nonpreferred shape. To determine the (ir)regularity of the RF, we computed an irregularity index, which was simply the maximum percentage that one would have to climb traveling from the global maximum to a local maximum when minimizing the height to be climbed but not the distance (Op De Beeck and Vogels, 2000). For neurons that showed at least two maxima in the RF profile (the highest being the global maximum and the others being local maxima), the RF irregularity represents the difference between the peak of the local maximum and the trough (the lowest response) located between the global maximum and the local maximum. The irregularity index was set to 0 if there was only one maximum in the RF profile. The RF steepness is the highest percentage decline in neural response per visual degree at the positions immediately adjacent to the global maximum.

We computed the maximum response to the worst shape normalized to the highest response of the neuron in the test (MNRW) and used this 
quantity as an indicator for the degree of position invariance: an MNRW $\leq 0.50$ suggests position invariance, MNRWs between 0.5 and 0.75 indicate weak position invariance, and MNRWs $>0.75$ indicate position-dependent responses.

\section{Results}

We recorded the activity of 142 single LIP neurons in the shape-tuning test (96 in monkey D and 46 in monkey E). Sixtyseven of these neurons were studied in the shape-position test, 23 in the saccademapping test, 26 in the target-cue test, and 23 in the memory saccade test.

\section{Shape-tuning test}

Shape tuning in single LIP neurons

A surprisingly large proportion of the neurons $(82 \%, 79$ of 96 in monkey D; 38 of 46 in monkey E) were significantly tuned to at least one of the shape dimensions in the stimulus set. The example neuron in Figure $2 \mathrm{~A}$ was tuned for all the dimensions tested in the stimulus set except aspect ratio (main effect of shape, $p<0.01$ ). The degree of tuning was very robust: for horizontal taper of the large shapes, for example, the average response varied from 4 to 41 spikes/s (arrows). Note that the two triangles at the extremes of the dimension are identical in surface area but simply rotated by $180^{\circ}$; hence, differences in the total luminance of the stimuli cannot account for the observed response differences. The neuron was strongly tuned for orientation, preferring the $45^{\circ}$ counterclockwise orientation, but orientation tuning alone could not explain the tuning for the other dimensions (see, for example, small shapes vertical taper and large shapes center-of-mass). Finally, the neuron in Figure $2 \mathrm{~A}$ exhibited some degree of size invariance: the shape preference was similar for the dimensions curvature (vertical and horizontal), horizontal taper, and orientation, but not for vertical taper and center-of-mass. The insert on the right shows the neural activity in the target-cue test: the neuron displayed strong spatially selective saccadic activity when the target appeared in the RF of the neuron, typical of LIP neurons.

In Figure $2 B$, a second example neuron is shown illustrating substantial tuning for multiple 2D shape dimensions (vertical and horizontal curvature, horizontal taper, aspect ratio, and orientation). As in the previous neuron, tuning strength was substantial (average response varied from 47 and 6 spikes/s for horizontal taper), but size invariance was virtually absent except for orientation. Lower-level features such as total luminance or orientation could not account for the observed shape tuning.

We analyzed the eye position traces before and during stimulus presentation. During the recording of the two example neurons, no significant changes in mean eye position occurred in the first $200 \mathrm{~ms}$ after stimulus onset ( $t$ test, NS), and the absolute differences in mean eye position were $<0.1^{\circ}$. Hence, differences in eye movements cannot account for the observed shape tuning of the neurons.

Overall, the ANOVA identified 104 of 142 neurons tested $(73 \%)$ having at least one significant main effect of shape (of which 96 also showed a significant main effect of size and 87 also showed a significant interaction between shape and size), and 13 of 142 neurons (9\%) with at least one significant interaction between size and shape but no significant main effects of shape or size. The former two groups together constituted our population of shape tuned neurons $(n=117)$. Small subsets of the neurons (14 of 142, 10\%) showed only significant main effects of size or no significant effect at all (11 of 142, 8\%).

\section{Population analysis}

The population histogram of all LIP neurons with significant shape tuning $(n=117)$ (Fig. $3 A)$ demonstrates that the average response difference between the best shape and the worst shape in the stimulus set was indeed substantial: except for a short transient response in the first $100 \mathrm{~ms}$ after stimulus onset, the firing rate during presentation of the worst shape was virtually identical to baseline, whereas the response to the best shape remained high throughout the duration of stimulus presentation. To quantify the degree of shape tuning, we calculated a shape-tuning index defined as STI $=$ (best - worst)/best, in which best is the response of the neuron to the preferred shape, and worst is the lowest response of the neuron along the same dimension as the best shape. The median STI measured 0.84 (Fig. $3 B$ ), corresponding to a more than sixfold average difference in response between the optimal shape and the least-preferred shape along a given dimension. In fact, it was not uncommon to observe inhibitory responses to the worst shape (STI $>1$ ). The distribution of STI was not significantly bimodal (Hartigan's dip test, $p=0.84$ ). The depth of selectivity indicates how broad the tuning for $2 \mathrm{D}$ shape 

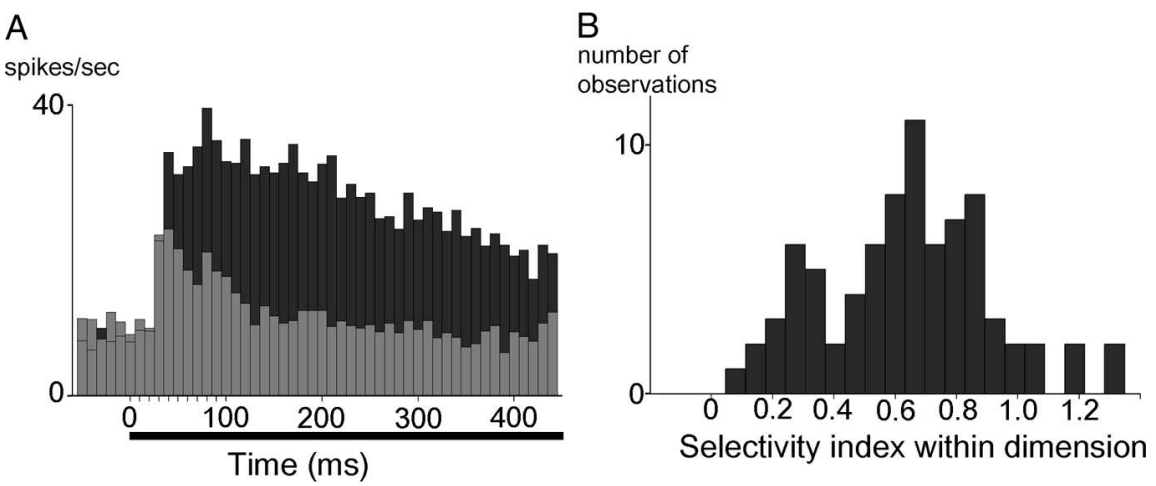

Figure 3. Population analysis of shape-tuning test. $\boldsymbol{A}$, Average response to the best shape (dark gray) and worst shape (light gray) for all 117 LIP neurons that were significantly tuned to 2D shape. Zero is the time of stimulus onset, and the horizontal bar indicates the duration of stimulus presentation. $\boldsymbol{B}$, Histogram of shape-tuning index ( $n=117)$.

was within our stimulus set. We computed an index for the selectivity depth defined as Sdepth $=(N-\mathrm{SUM} / \mathrm{MAX}) /(N-1)$, in which $N$ is the number of conditions, SUM is the sum of the responses, and MAX is the maximum response (Rainer et al., 1998). The index is 1 if the neuron responds to only one stimulus, and 0 if the neuron responds to all stimuli. The mean selectivity depth measured $0.63 \pm 0.02$, indicating relatively broad tuning. Eye position traces were analyzed for each neuron with significant shape tuning. The number of neurons having significant changes in mean eye position before stimulus compared with after stimulus onset $(0-200 \mathrm{~ms})$ was not higher than expected by chance $(7 \%)$.

\section{Latency of the neural response}

The latency of the population response was remarkably short. For both the best and the worst shape, the population activity rose above baseline as early as $37 \mathrm{~ms}$ after stimulus onset (Poisson test, $p<0.01)$. The first bin in which the population response to the best shape differed significantly from the response to the worst shape ( $t$ test, $p<0.05$ ) occurred between 40 and $50 \mathrm{~ms}$ after stimulus onset. In our population of 117 tuned LIP neurons, the median latency was $65 \mathrm{~ms}$, but a substantial fraction of the neurons (19\%) became active as early as $50 \mathrm{~ms}$ after stimulus onset. The rapid emergence of response differences argues strongly against any explanation in terms of eye movement planning and/or attention processes but rather suggests that the observed tuning for $2 \mathrm{D}$ shape dimensions is a fast bottom-up process reaching LIP not long after the visual information has arrived in primary visual cortex.

\section{Effect of stimulus size}

For the large majority of LIP neurons in our sample of shapetuned neurons ( 80 of $117,68 \%$ ), the best shape belonged to the $5^{\circ}$ shape set. Because every neuron was tested with both sizes, this observation provides an unbiased estimate of the optimal stimulus size for LIP neurons, at least within the size range tested in this experiment. On average, the LIP response to the best shape with the preferred size equaled $33.7 \pm 1.8$ spikes/s, but the corresponding shape of the nonpreferred size evoked an average of only $10.9 \pm 1.2$ spike/s. Moreover, the large majority of the shape-tuned neurons (100 of 117, 84\%) showed a significant interaction between shape and size. These data indicate that simple 2D shapes are effective stimuli for LIP neurons, but the effect of stimulus size on the neural response was substantial.

The example neuron in Figure $2 B$ clearly demonstrates that the shape preference was not preserved for different stimulus sizes. For only a small fraction of the neurons tested (11 of $117,9 \%$ ) did the two or three of the most effective shapes of the nonpreferred size also correspond to the three best shapes of the preferred size. To further quantify the degree of size invariance, we ranked the net responses of each neuron according to the shapes with the preferred size and then applied the same ranking to the responses of the shapes with the nonpreferred size (Fig. 4). The slope of the curve for the nonpreferred size, albeit significantly $>0$, was much shallower $(-0.18)$ than the slope for the preferred size $(-0.97)$. Hence, shape preference in LIP neurons was substantially reduced when these were tested with another stimulus size. The degree of size invariance, however, differed between the shape dimensions in the stimulus set (Fig. 5). Size invariance was strongest for the combination of aspect ratio and orientation and weakest for the dimensions curvature and taper: the slope of the ranking curve for the nonpreferred size was equal to 23 and $24 \%$ of the slope for the preferred size for orientation + aspect ratio and center-of-mass, respectively, compared with only $9 \%$ for curvature and $11 \%$ for taper (ANOVA, $p=0.01$ ). Thus, the ranking analysis indicates that size invariance in LIP neurons is incomplete and mainly present for relatively simple shape features such as aspect ratio or orientation.

A second measure of size invariance is given by the separability index. The mean separability index across our population of LIP neurons was $0.70 \pm 0.02$, which is substantially lower than values reported for visual area V4 (0.87) (Brincat and Connor, 2004). Given the results of the ranking analysis, however, we believe that the separability index may overestimate the degree of size invariance.

\section{Shape-position test}

In total, 67 neurons were studied further with preferred and nonpreferred shapes at multiple positions in the contralateral hemifield: 54 neurons were tested at 35 positions, and 13 neurons were tested at 11 different positions. The vast majority of the neurons tested (63 of 67) were shape tuned according to the shape test.

\section{Coding of shape and/or position: single-neuron examples}

The neuron in Figure $6 A$ (the same as in Fig. $2 B$ ) fired vigorously to a $5^{\circ}$ shape tapered in the horizontal direction toward the right but only weakly to the opposite taper. The strongest response was observed at $4^{\circ}$ eccentricity on the horizontal meridian, but the neuron also fired at more than half of its maximum response at nine additional positions extending $8^{\circ}$ in the horizontal direction and $4^{\circ}$ in the vertical direction. The RF size, measured using the preferred shape (defined as the square root of the area within the $50 \%$ contour), equaled $5.9^{\circ}$. The response to the nonpreferred shape, in contrast, never exceeded $30 \%$ of the maximum response of the neuron. Hence, this example neuron preserved its selectivity over different positions in the frontoparallel plane. Because the two stimuli were exactly matched in terms of lowlevel features such as luminance and orientation, we can consider this neuron to be truly shape selective, in agreement with the results of Sereno and Maunsell (1998). Note, however, that this neuron barely responded to small shapes along the same dimension in the shape-tuning test and, hence, showed no size invari- 


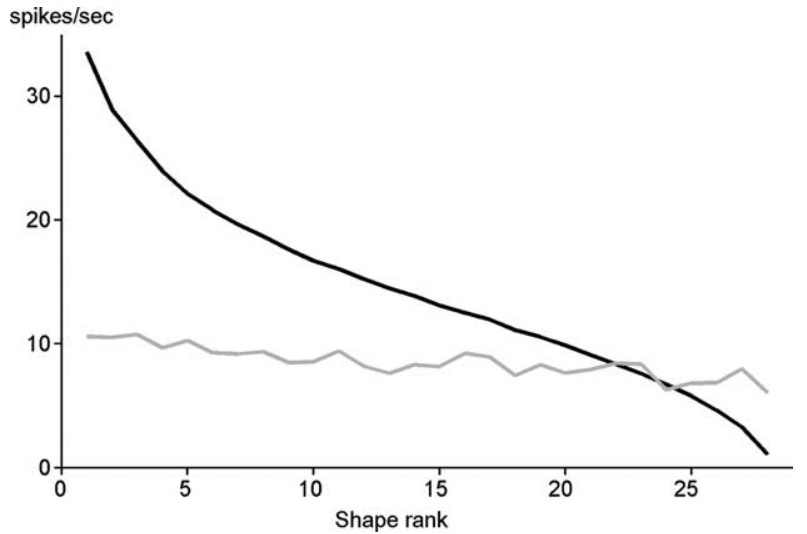

Figure 4. Size invariance in the shape-tuning test for those LIP neurons that responded significantly to both sizes $(n=93)$. Red, Average ranked responses to the shapes with the preferred size. 1, Shape to which the neuron showed the highest response (best shape); 28 , shape to which the neuron showed the weakest response (worst shape). Blue, Average response to the corresponding shapes with the nonpreferred size.
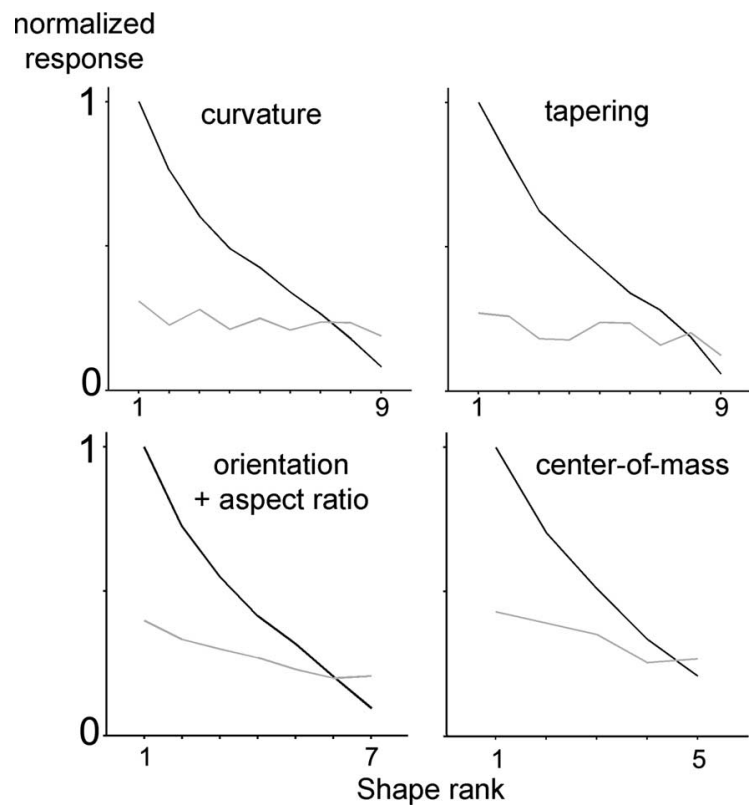

Figure 5. Size invariance per dimension $(n=93)$. Average ranked normalized responses to the shapes with the preferred size (red) and average normalized responses to the corresponding shapes with the nonpreferred size (blue). curvature, Vertical and horizontal curvature; tapering, vertical and horizontal tapering.

ance. The neuron in Figure $6 B$, conversely, exhibited a fairly small RF $\left(2.6^{\circ}\right)$ located at $2^{\circ}$ eccentricity in both the vertical and the horizontal direction. In fact, at only two positions, spaced $2^{\circ}$ apart, did the neural response to the horizontal bar exceed $50 \%$ of its maximum. The largest response to the nonpreferred shape (a square) was $43 \%$ of the maximum response; hence, this neuron can also be considered 2D shape selective, albeit with a surprisingly small RF. Overall, $19 \%$ of the neurons in our sample were entirely position invariant, i.e., the highest response to the nonpreferred shape never exceeded $50 \%$ of the maximum response of the neuron at any position tested (MNRW $<0.50$ ) (see Materials and Methods).

The RF of the LIP neuron in Figure $6 C$ appeared to be more complex: the RF contained two maxima, one up and to the right and a second one down and to the right. RF size equaled $8.9^{\circ}$,
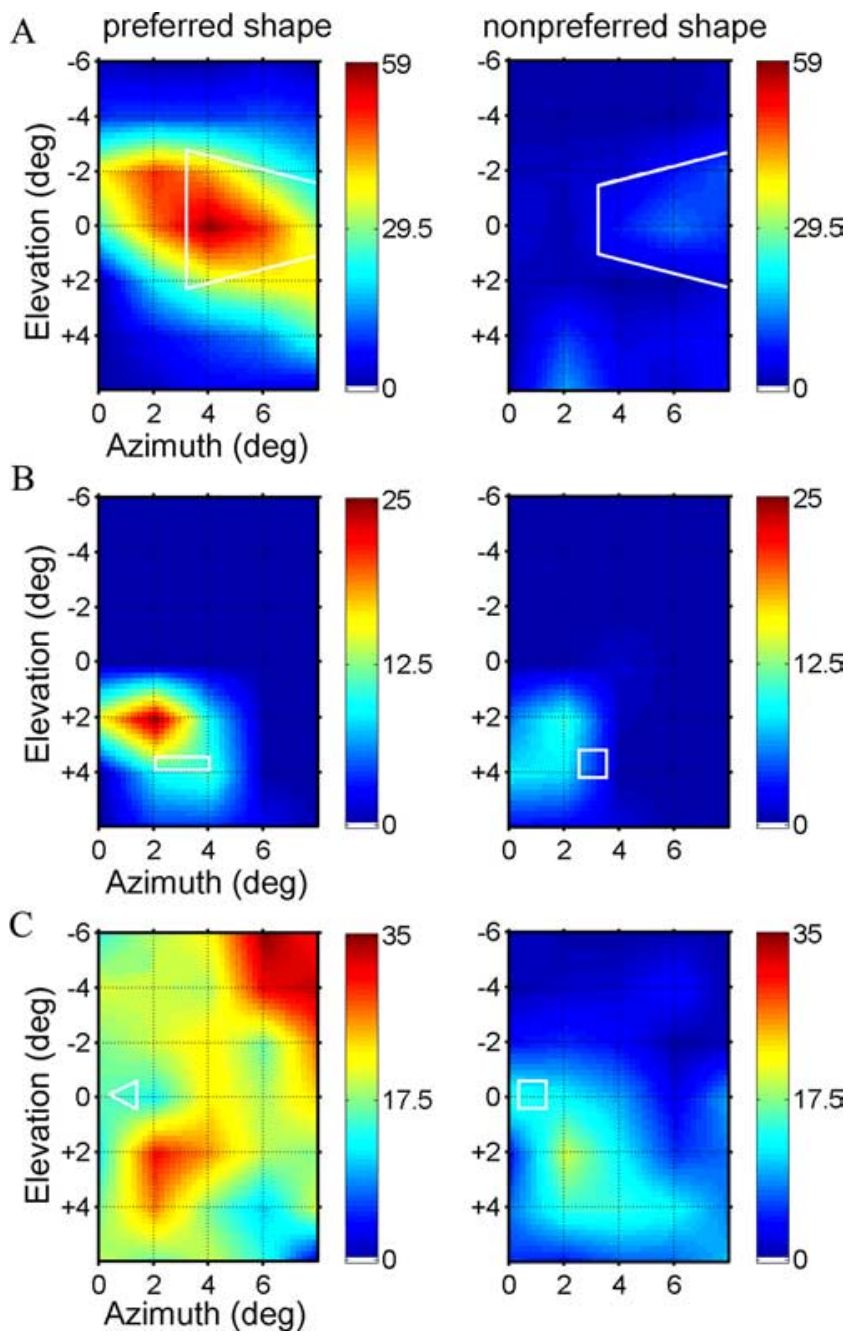

Figure 6. Shape-position test: position-invariant neurons. The colors indicate the net response of the neuron plotted as a function of horizontal (azimuth) and vertical (elevation) position of the shapes. The fixation point is at $(0,0)$. Shapes were presented at each cross-section in the grid ( $2^{\circ}$ spacing). The left column represents the responses to the preferred shape, and the right column represents the responses to the nonpreferred shape. The white contours indicate these shapes at the position tested in the shape-tuning test. $\boldsymbol{A}$, Position-invariant example neuron with a large and homogeneous RF. $\boldsymbol{B}$, Position-invariant example neuron with a small RF. C, Weakly position-invariant example neuron with an heterogenous RF.

which was above the 90th percentile in our sample of neurons. Intriguingly, the neuron also responded at the second local maximum to the worst shape at $57 \%$ of the overall maximum response but gave no response at all at the global maximum of the $\mathrm{RF}$ in the upper right quadrant. Consequently, if this neuron were to have been tested only in the upper right quadrant $\left(\mathrm{a} 6 \times 6^{\circ}\right.$ area in which the neuron was essentially silent during presentation of the square), it would have appeared as perfectly position invariant, but it is not. At best, we can regard this type of neuron as weakly invariant. For $21 \%$ of the neurons, the largest response to the worst shape fell between 50 and $75 \%$ of the maximum response of the neuron and, hence, were labeled weakly position invariant.

The other extreme of the spectrum, a position neuron, is shown in Figure 7A. This neuron was not significantly tuned to any of the shape dimensions in the shape-tuning test. The RF was large and relatively homogeneous for both stimuli used. Despite being strongly activated by the presentation of small shapes inside the RF, this type of neuron does not provide any information 

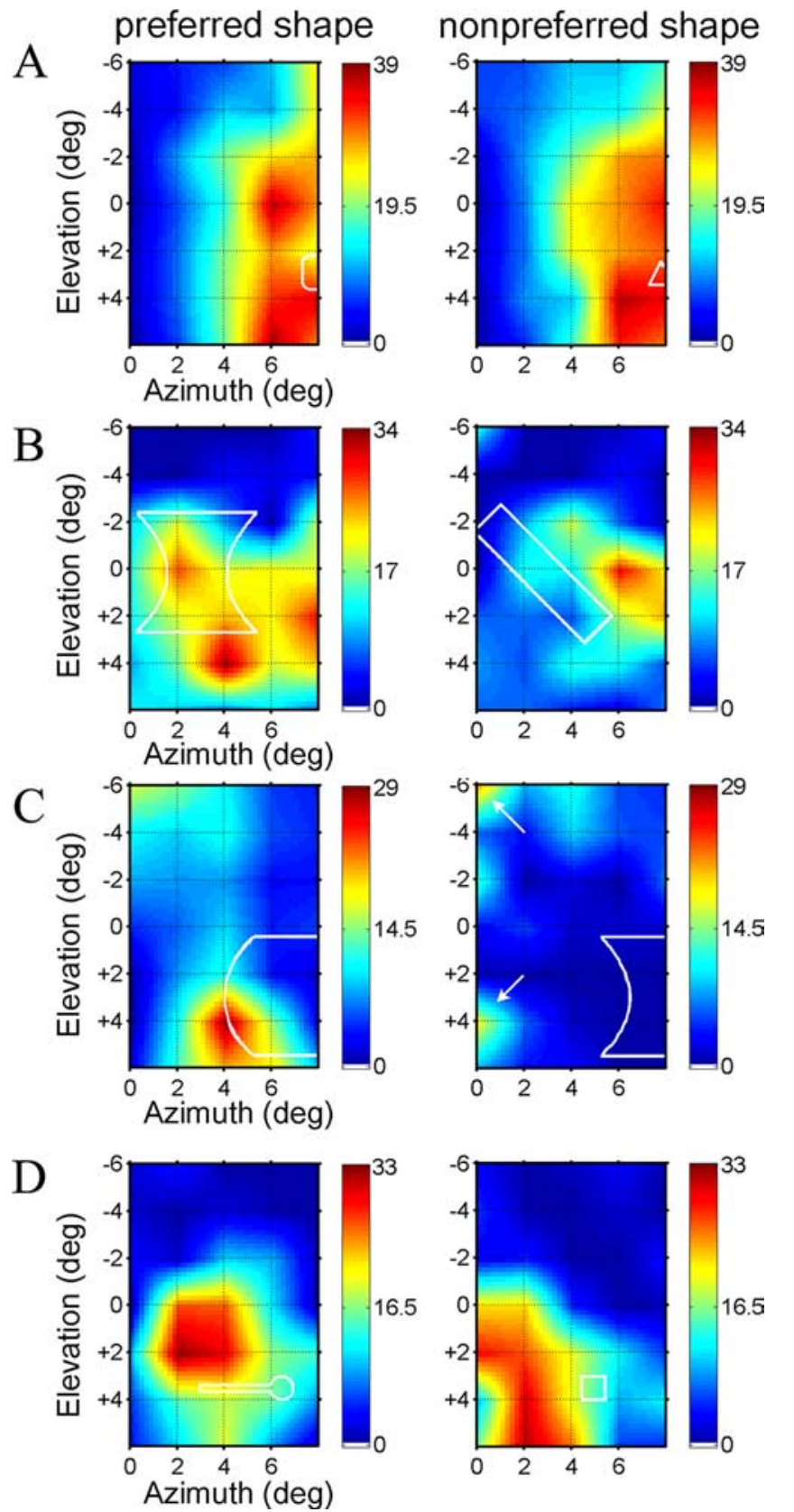

Figure 7. Shape-position test: position-dependent neurons. $\boldsymbol{A}$, Example neuron that was not significantly tuned in the shape-tuning test and showed similar RF profiles for both shapes tested. $\boldsymbol{B}$, Example neuron showing significant response differences in the shape-tuning test but equally large responses to the nonpreferred shape in the shape-position test. $\boldsymbol{C}$, Example neuron with multifocal RF for the two shapes tested. Arrows indicate the two positions where the neuron fired strongly to the nonpreferred shape. $\boldsymbol{D}$, Example neuron with partially overlapping but heterogeneous RF profiles for preferred and nonpreferred shape. Same conventions as in Figure 6.

about shape but, rather, signals where in the visual field salient stimuli appear. A second example neuron is illustrated in Figure $7 B$. In the shape-tuning test, the neuron responded strongly to the $5^{\circ}$ shape with concave curvature and only weakly to the oriented bar. The results of the shape-position test confirmed this apparent selectivity: robust responses to the best shape and weak or absent responses to the worst shape in an area lying between 2 and $4^{\circ}$ along the horizontal direction in the lower quadrant. Presenting the worst shape at $6^{\circ}$ eccentricity along the horizontal meridian, however, evoked strong responses comparable with the best shape responses ( $89 \%$ of the maximum response). The results of the shape-position test therefore suggest that, in the shape-tuning test, the alignment of the stimulus with the RF of the neuron was suboptimal for the oriented bar, which produced a spurious shape selectivity. A $2^{\circ}$ shift in position along the horizontal meridian was sufficient to activate the neuron almost as strongly as the presentation of the best shape. Hence, this type of neuron is not position invariant but merely signals where a stimulus appears in the visual field. In our population of neurons, $40 \%$ of the neurons showed this type of response behavior (position neurons).

A more puzzling case is illustrated in Figure 7C. The RF measured with the best shape appeared relatively restricted: the LIP neuron responded well to the $5^{\circ}$ shape with convex curvature at three positions in the lower right quadrant, whereas the response to the worst shape in that region was negligible. Surprisingly, two other positions on the vertical meridian, at $6^{\circ}$ up and at $4^{\circ}$ down (arrows), yielded robust responses to the worst shape (72 and $62 \%$ of the maximum response, respectively). The response at these two positions differed significantly from all other positions in the immediate vicinity ( $p<0.01, t$ test), although there was no overlap with the hot spot of the RF as measured with the best shape. The upper visual field position, but not the one in the lower visual field, did coincide with a weak $57 \%$ of the maximum response) second local maximum in the RF of the best shape. In a manner similar to the neuron in Figure $6 C$, shape preference was preserved over a fairly large range of positions spanning an $8 \times 6^{\circ}$ region. Because some positions outside this area evoked significant responses to the worst shape, however, this neuron is not entirely position invariant. Figure $7 D$ shows a second example neuron that yielded spurious shape selectivity in the shapetuning test attributable to a misalignment of the worst shape just outside the RF of the neuron. Although there was considerable overlap between the RFs of the two shapes tested, the strongest response to the "worst" shape (the square) was observed at a position where the "best" shape did not activate the neuron at all (i.e., $6^{\circ}$ down and $2^{\circ}$ to the right). Hence, this neuron exhibited highly heterogenous but overlapping RF profiles for the two shapes used in the test. To verify that our recordings were not inadvertently contaminated by a second neuron, we stored the raw signal recorded during the experiment for off-line analysis. The signal-to-noise ratio for the neuron in Figure $7 D$ was 5.5:1, rendering such contamination extremely unlikely. Neurons showing a substantial response to the worst shape ( $>50 \%$ of the maximum response) at a position not overlapping the global maximum of the RF as measured with the best shape were termed multifocal neurons (either weakly invariant as the neuron in Fig. $7 C$ or not invariant as in Fig. 7D) and accounted for 19\% of the neurons in our population. This type of neuron may extract certain shape features in the image, possibly within a restricted region of space, but the information that these neurons might provide to the next level in the hierarchy of visual areas is highly ambiguous with respect to both shape and position.

\section{Population analysis of the position test}

The average RF in our population of LIP neurons is plotted in Figure 8 . The center-of-mass of this average RF was located at $4^{\circ}$ along the horizontal meridian in the lower visual field, which indicates a slight overrepresentation of neurons responding to the lower hemifield in our sample. The average RF as determined with the nonpreferred shape was located at a similar position but was much smaller than the RF as determined using the preferred shape. 
Across our population of LIP neurons tested $(n=67)$, the MNRW averaged 0.75. The distribution of the MNRW, although significantly different from the normal (Jarque-Bera test for normality, $p<0.05$ ), was not significantly bimodal (Hartigan's dip test, $p=0.75$ ). Hence, there is little evidence for a separate subgroup of position-invariant LIP neurons but, rather, a continuum from positioninvariant to position-dependent response patterns.

RF size measured with the best shape averaged $5.3^{\circ}$ (percentile $10,1.5^{\circ}$; percentile $90,9.2^{\circ}$ ) in our population of neurons compared with $3.2^{\circ}$ when measured with the worst shape. This measurement, however, is likely to be an underestimation of the real RF size: our range of positions was limited to $12^{\circ}$ in the vertical direction and $8^{\circ}$ in the horizontal direction, and, for all LIP neurons tested, the RF extended out to the edge of the tested region (Figs. 6C, 7A). The steepness of the RF was estimated by calculating the largest percentage decline in response per visual degree starting from the position where the neuron gave the strongest response to the stimulus. For example, the RF of the neuron in Figure $7 B$ had a steepness of 0.40 for the best shape and 0.30 for the worst shape. In other words, shifting the best shape by as little as $2^{\circ}$ (down in this case) reduced the response by $80 \%$. On average, RF steepness equaled $0.27 \pm 0.01$ for the best shape and $0.20 \pm$ 0.01 for the worst shape (Fig. 9A). The sharp decline in response at the edge of the RF can explain why we measured spurious shape selectivity, even with $1^{\circ}$ shapes.

We quantified the irregularity of the RF based on the normalized responses to the best and the worst shape for all neurons that showed at least two maxima in the RF profile: the global maximum was the point in the visual field with the highest response, and all other points where the response peaked were labeled local maxima. The RF irregularity was calculated as the difference between the peak of the local maximum (provided it existed) and the trough (the lowest response) located between the global maximum and the local maximum (Op de Beeck and Vogels, 2000). The mean irregularity was $0.13 \pm 0.02$, but a sizeable proportion of the neurons showed highly irregular RFs (Fig. 9B): for $20 \%$ of the neurons, the difference between the local maximum and the trough was $30 \%$ of the response or more, which is considerably larger than in data reported for IT (Op de Beeck and Vogels, 2000).

For each neuron that responded at $>50 \%$ of the maximum response to the nonpreferred shape $(n=43)$, we calculated the position of the center-of-mass of the RF for both shapes. The center of the RF as measured with the preferred shape was generally located close to the center of the RF as measured with the nonpreferred shape (Fig. 9C): the distance between the RF cen-

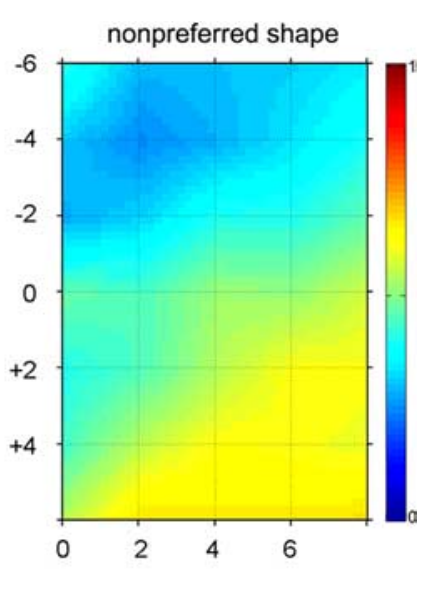

Figure 8. Average RF for all LIP neurons tested in the shape-position test ( $n=67)$. Same conventions as in Figure 6 .

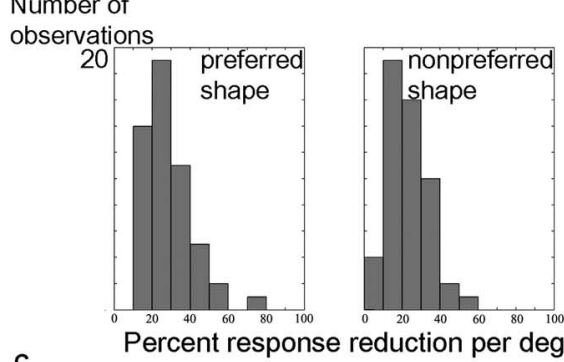

Number of

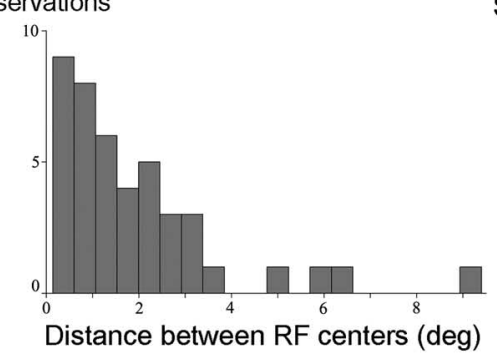

Figure 9. RF properties in shape-position test $(n=50)$. $\boldsymbol{A}$, Histograms of RF steepness for preferred (left) and nonpreferred (right) shape. $\boldsymbol{B}$, Histogram of the RF irregularity index (see Materials and Methods). $\boldsymbol{C}$, Histogram of the distance between RF hest response to the nonpreferred shape (ordinate) versus the shape-tuning index in the shape-tuning test (abscissa). Diagonal line indicates equal selectivity in both tests.

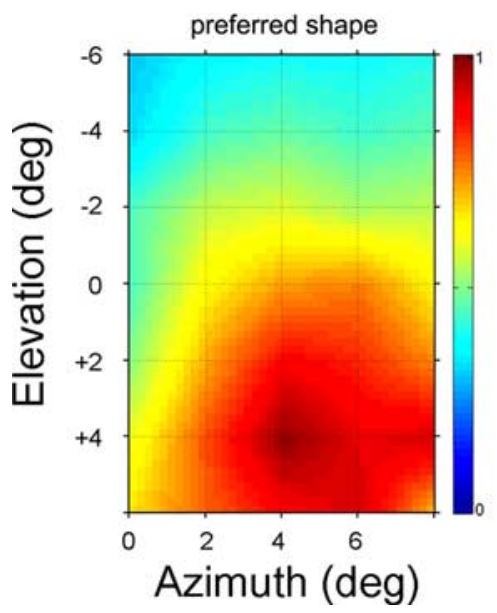

\section{B}

Number of

observations

60

D

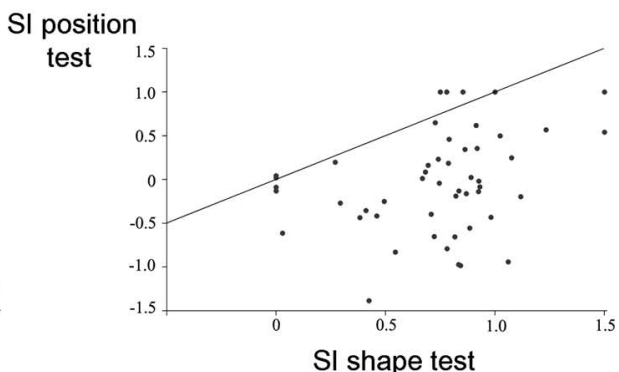

ters was $<3^{\circ}$ in $>80 \%$ of the neurons. Nevertheless, we observed considerable differences between the preferred shape RF and the nonpreferred RF (Fig. $7 B, C$ ): the median percentage overlap in the RF profiles for both shapes was only $60 \%$. These results indicate that the RF profile of LIP neurons can depend strongly on the stimulus used to map the RF.

Finally, we compared the shape-tuning index (determined in the shape-tuning test) with the selectivity in the shape-position test at the position where the neuron showed the strongest response to the nonpreferred shape (Fig. 9D). For neurons that did not respond to the nonpreferred shape at any position, the selectivity index was set to 1 . Almost all data points are located below the diagonal, and many points are centered around or even below 0 on the ordinate (which is the selectivity in the shape-position test). These results clearly demonstrate that the degree of shape 
A

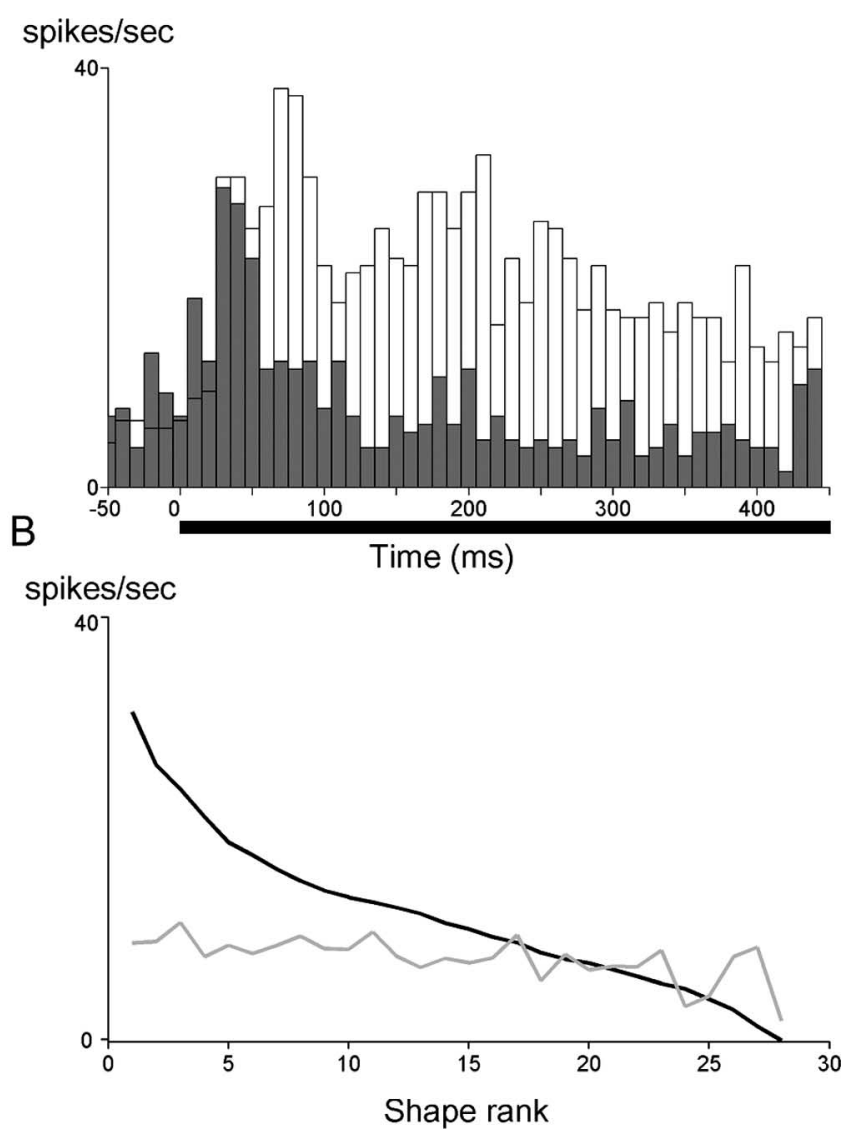

Figure 10. Position-invariant neurons $(n=13)$. $\boldsymbol{A}$, Population response of positioninvariant neurons to the best and the worst shape. Same conventions as in Figure $3 A$. $\boldsymbol{B}$, Size ranking for position-invariant neurons. Red, Average ranked responses to the shapes with the preferred size. 1, Shape to which the neuron showed the highest response (best shape); 28, shape to which the neuron showed the weakest response (worst shape). Blue, Average response to the corresponding shapes with the nonpreferred size.

tuning is strongly overestimated if one presents the stimuli at a single position in the visual field.

Figure $10 \mathrm{~A}$ shows the population response to the best and the worst shapes in the shape-tuning test for those neurons that proved to be position invariant in the shape-position test $(n=$ 13). Although the response latency $(30-40 \mathrm{~ms})$ and the magnitude of the response difference are comparable with the entire population of LIP neurons tested, the latency of the response difference is markedly longer: this subpopulation of neurons starts to signal the difference between the shapes only after 60-70 $\mathrm{ms}$. This latency is comparable with selectivity latencies in ventral areas such as V4 (Mysore et al., 2006). In Figure $10 \mathrm{~B}$, the ranked average responses are shown for the preferred and the nonpreferred size. Similar to the total population, the degree of size invariance was very limited in this subpopulation of positioninvariant neurons.

\section{Saccade-mapping test}

Basic properties of LIP neurons in the saccade task

Approximately $90 \%$ of the neurons tested in the saccade tasks (21 of 23 in the saccade-mapping test and 18 of 21 in the target-cue test) showed spatially selective saccadic activity. In the saccademapping test, the RF center was located at an average of $7^{\circ}$ eccentricity (percentile $10,4.8^{\circ}$; percentile $90,8.9^{\circ}$ ) compared with $4^{\circ}$ for the shape RF. Firing rate during saccades toward the RF was robust: the mean response from 80 to $400 \mathrm{~ms}$ after target onset averaged $36.5 \pm 8.3$ spikes/s in the saccade-mapping test and $24.8 \pm 14.4$ in the target-cue test.

In the target-cue test, the average response in the target-in/ cue-out condition (19.5 spikes/s) was very similar to the response in the target-out/cue-in condition (19.2 spikes/s, NS). Hence, the LIP neurons in our sample responded equally well when the stimulus in the RF was a saccade target or a go cue.

Although most neurons ( 15 of 23, 65\%) exhibited significant delay period activity in the memory saccade test, the level of delay activity was rather modest: the mean activity from 320 to $720 \mathrm{~ms}$ after target onset averaged only $60 \%$ of the initial response (80$320 \mathrm{~ms}$ after target onset), which is low compared with other studies (Janssen and Shadlen, 2005). The presaccadic enhancement in neural activity, typical of LIP neurons, averaged 6.4 spikes/s, and 17 of 44 neurons tested (39\%, 10 of 21 in the targetcue task and 7 of 23 in the saccade-mapping task) showed significant presaccadic increases in activity. Overall, the properties of the LIP neurons in our sample are in agreement with those reported in previous studies (Barash et al., 1991; Pare and Wurtz, 1997; Shadlen and Newsome, 2001).

\section{RF profile determined with shapes and saccades}

Eleven neurons were studied in both the shape-position test and the saccade-mapping test. The $x$ and $y$ coordinates of the maximum neural response matched relatively well in the two tests: the 2D correlation coefficient between the $x$ and $y$ coordinates of the RF maxima in the saccade-mapping test and in the shapeposition test was $0.72(p<0.01)$. The RF of the neuron in Figure $11 A$, for example, mapped with the preferred shape was centered at $6^{\circ}$ eccentricity on the horizontal meridian, and the saccadic RF was located at a comparable position. When tested with the nonpreferred shape (an elongated vertical bar), however, the strongest responses were elicited in the lower right quadrant. In Figure $11 A$, the endpoints of the nonpreferred shape have been superimposed on the RF map. Thus, it becomes clear that the neuron fired twice as strongly when the top part of the elongated bar appeared in the center of the RF as when the bottom part of the bar was presented in the RF (ANOVA, $p<0.01$ ). These results suggest that LIP neurons that do not display complete positioninvariant shape selectivity can provide some shape information: these neurons may signal the appearance of interesting shape parts at a particular location in space, possibly the target of an upcoming saccade. The elongated bar may have two potential saccadic target points (remarkable points), the upper end point and the lower end point, whereas the preferred shape (the horizontally tapered shape) may have been compact enough to represent an entire saccade target. A second example neuron (Fig. $11 B$ ) responded whenever the lower edge of the preferred shape appeared at $4.5-10.5^{\circ}$ eccentricity on the vertical meridian, matching closely with the saccadic RF. For other LIP neurons, however, the saccadic RF differed substantially from both the preferred and the nonpreferred shape RF (Fig. 11C), and, for these, no common region in space could be identified for the shape and saccadic RFs. Remarkably, almost half of the neurons tested ( 5 of 11) showed both significant shape responses at the foveal position and an eccentric saccadic RF (e.g., the nonpreferred shape RF in Fig. 11C). These results strongly suggest that the spike rate of LIP neurons can be substantially influenced by presenting shapes, and possibly other visual stimuli, outside the saccadic RF. 


\section{Recording area}

LIP occupies a large part of the lateral bank of the IPS, extending up to $10 \mathrm{~mm}$ in the anteroposterior direction and $8 \mathrm{~mm}$ mediolaterally (Andersen et al., 1990; Durand et al., 2007). Moreover, considerable uncertainty exists regarding the exact boundaries of LIP with area AIP anteriorly and the caudal intraparietal area posteriorly (Lewis and Van Essen, 2000; Murata et al., 2000; Borra et al., 2008). Hence, it is important to verify that the recording positions of different single-cell studies are similar. We recorded neurons, most of which showed saccadic activity, in the lateral bank of the IPS between Horsley-Clark coordinates 3P and 7P and between 9L and 15L. These coordinates compare well with those reported by Lehky and Sereno (2007): P1 to $\mathrm{P} 6$ and $10 \mathrm{~L}$ to $12 \mathrm{~L}$. Figure $1 B$ illustrates the recording positions projected onto the flat map of the IPS used by Durand et al. (2007). In both animals, we recorded in the anterior part of LIP, posterior to the boundary of LIP as determined by the activations evoked by saccades versus their visual control. We made several penetrations into area AIP (at more anterior grid positions between $2 \mathrm{P}$ and $1 \mathrm{~A}$ ) but did not find any responses to our simple $2 \mathrm{D}$ shapes. Monkey D was also used in a separate experiment using three-dimensional (3D) shapes (S. Srivastava, G. A. Orban, and P. Janssen, unpublished observations): only 2 $\mathrm{mm}$ separated the recording positions with $3 \mathrm{D}$ shape-selective neurons from the most anterior recording position in the present study. We did not observe any responses to these 3D shapes in LIP, but, because the stimuli in the present study were only presented at the fixation point, we cannot draw any firm conclusions from these observations. Note that we observed no differences in neural selectivities for 2D shape between more anterior and posterior recording positions within the range explored in LIP.

\section{Discussion}

We investigated the tuning of LIP neurons for shape dimensions important for grasping. Many LIP neurons were tuned to one or more shape dimensions, and the tuning was robust and evolved remarkably rapidly. The shape preference of LIP neurons was generally not invariant over stimulus transformations. Many LIP neurons displayed spurious shape selectivity attributable to interactions between the stimulus position and the RF profile.

\section{Joint coding of shape and position by LIP neurons}

Sereno and Maunsell (1998), using just eight small shapes in their stimulus set, reported many LIP neurons (57\%) showing significant response differences (average response ratio, 2.5:1). These authors argued that the observed selectivity is unlikely to arise from accidental interactions between shape features and RF profiles, because LIP RFs are typically large and homogeneous. A
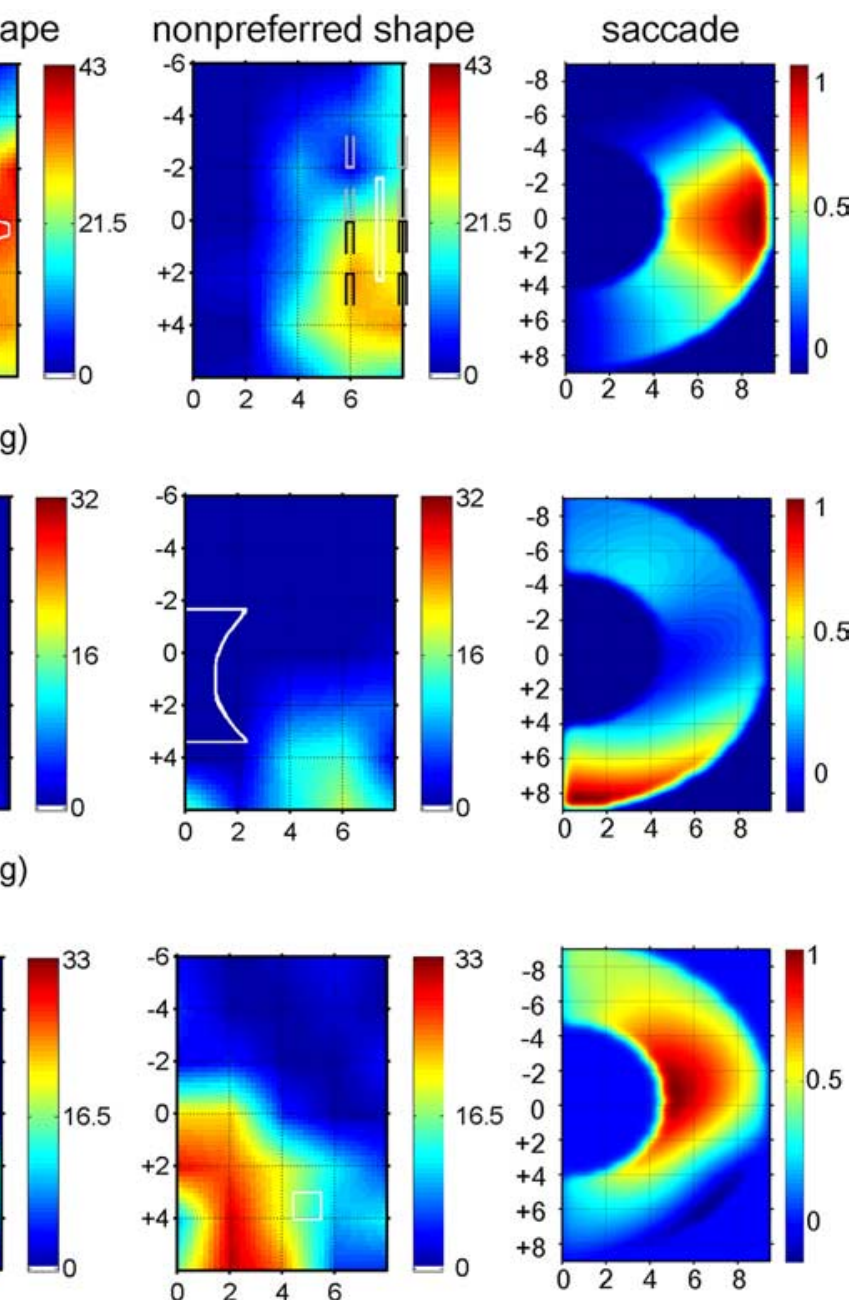

Figure 11. Saccade-mapping test and shape-position test. The left column represents the responses to the preferred shape, the middle column the responses to the nonpreferred shape, and the right column the responses in the saccade-mapping test Presteffive positions (top) are indicated in gray. White contours represent the shape position in the shape-tuning test. $\boldsymbol{B}$ Position-invariant example neuron where the RF profile for saccades matches the RF profile for the best shape. C, Example neuron in which the RF profile is different for best shape, worst shape, and saccade test. Same conventions as in Figure 6.

small number of neurons were tested with the same stimulus set at a second location within the RF, producing good agreement between the shape preferences at the two locations.

With a more extended shape set and two sizes, we observed stronger shape tuning (median response ratio, 6:1) in an even larger proportion of LIP neurons (82\%). In the Sereno and Maunsell (1998) study, however, the monkeys were trained to make saccades to the shapes in a delayed match-to-sample task. Although the shape selectivity was tested during passive fixation, the observed shape selectivity in LIP might have been induced by the extensive training phase, or the animals could have been covertly planning saccades to (different parts of) the shapes. We replicated the shape tuning of LIP neurons in monkeys that had never made eye movements to any of the shapes in the stimulus set. Furthermore, the latency of the differential response in our population of LIP neurons was too short to allow the programming of saccades. Hence, shape tuning in LIP is most likely not the result of training in, nor the covert planning of, saccades toward shapes. 
A second difference between our study and the one by Sereno and Maunsell (1998) lies in the number of positions tested. We tested the central $8^{\circ}$ of the contralateral visual field, where most LIP RFs are located (Ben Hamed et al., 2001). A central finding was that it is crucial to test a large number of positions because many LIP neurons preserved their shape preference over a number of positions but failed the test at other positions. Asymmetric RFs were described previously in LIP (Ben Hamed et al., 2001) and in dorsal the medial superior temporal area (Lagae et al., 1994). Spurious shape selectivity can also be observed in the absence of RF asymmetry provided the RF profile for the two shapes tested is heterogenous (Fig. $7 A, D$ ). Similarly, the saccadic RF may differ substantially from the shape RF (Fig. 11C). Clearly, the saccade target could be considered as a third shape used to map the RF.

The irregular structure and the heterogeneity of the RF profile for different stimuli in many LIP neurons make it particularly difficult to assess stimulus selectivity without extensively mapping the RF. The spurious shape selectivity in our subset of LIP neurons resembles the apparent optic flow sensitivity in the middle temporal area MT/V5 neurons, which can be entirely explained by a selectivity for the lower-order feature translation (Lagae et al., 1994). In our study, however, we observed the entire spectrum between position-invariant and position-dependent responses. The functional significance of the joint coding of shape and position is unclear at present. We can only speculate that most LIP neurons do not provide an abstract representation of $2 \mathrm{D}$ shape but, rather, a combination of shape features and lower-order features such as position, possibly for the purpose of making eye movements to the most informative points on the object (Andersen and Buneo, 2002). Ideally, LIP neurons should be tested with a large number of shapes and positions, but the time constraints inherent to single-cell recordings made such a test impractical.

\section{Latency of the LIP responses}

We observed remarkably short response and selectivity latencies, in agreement with Bisley et al. (2004). These short latencies argue against any role for saccade programming or spatial attention in the shape tuning reported here. The observation that "genuine," position-invariant shape selectivity arises $30 \mathrm{~ms}$ later in the response indicates that the rapid neural responses originate from neurons signaling mainly position in space, not shape per se. The longer latencies of position-invariant neurons leave open the possibility that LIP receives shape information from ventral area V4, with which it is interconnected (Lewis and Van Essen, 2000). Alternatively, position invariance may be computed locally within area LIP based on inputs from area V3A (Nakamura et al., 2001). In this view, the incomplete position invariance of LIP neurons represents a within-modality analog of the partial shifts of visual RFs observed in neighboring area ventral intraparietal area (VIP) (Avillac et al., 2005). Tactile RFs of multimodal VIP neurons are invariant in head-centered coordinates, but their visual RFs frequently show an incomplete shift between eyecentered and head-centered coordinates. A model of optimal multisensory integration (Deneve et al., 2001) using recurrent basis function networks is capable of computing the most likely location of an object and provides an attractive account of the observed phenomena in VIP. Intriguingly, the model correctly predicted that (invariant) head-centered VIP neurons exhibit longer visual latencies than (not invariant) eye-centered neurons: short-latency responses reflect the initial state of the network (in this case, the purely eye-centered visual input), whereas neurons with longer latencies reflect the state of the network after several iterations. In our study, the longer latencies of position-invariant LIP neurons may be the result of several iterations in a recurrent network of activity starting with purely position-dependent responses.

\section{Comparison with IT}

Within the limits of our test procedure, we can compare some basic RF properties of LIP and IT (Op de Beeck and Vogels, 2000). The center of the mean RF in LIP was not at the fovea as in IT but $4^{\circ}$ away from the fovea in both the horizontal and vertical direction. For the great majority of the neurons ( 44 of 54, 81\%), however, the RF encroached on the central $2^{\circ}$ of the visual field. The RF profile of most IT neurons is symmetric and regular, whereas in LIP considerable RF irregularity was observed. Mapping the RF of IT neurons with different shapes generally yields similar RF profiles, whereas a considerable fraction (32\%) of the LIP neurons in our study showed differences in RF position exceeding $2^{\circ}$. Note that we observed a slight overrepresentation of the lower visual field in our sample of neurons, possibly attributable to an oversampling of the more anterior recording positions (Ben Hamed et al., 2001).

\section{Size invariance}

LIP neurons showed exquisite sensitivity to stimulus size. In fact, size invariance was virtually absent at the population level except for the dimensions orientation and aspect ratio. Sereno and Maunsell (1998) used a 50\% increase in size, whereas the size variation was $500 \%$ in our study. IT neurons, however, tolerate size changes over a much larger range (Ito et al., 1995; Op de Beeck and Vogels, 2000). The observation that even positioninvariant LIP neurons are not size-invariant points to the importance of stimulus size in LIP. Surely, if visual information regarding object shape in LIP is ultimately used to program the appropriate grasp, it is hardly surprising that stimulus size is not discarded by LIP neurons. The lack of size-invariant shape selectivity suggests once more that the shape representation in area LIP differs considerably from the shape representation in IT.

\section{Saccadic activity and shape tuning}

Almost all of our LIP neurons exhibited spatially selective saccadic activity. Hence, shape tuning can be observed in LIP neurons identified using widely accepted criteria (Barash et al., 1991). Our results suggest that LIP neurons are capable of rapidly signaling the location of salient objects or potential gaze targets that come into view complemented by some rudimentary form information, a signal that can be exploited by other oculomotor neurons to efficiently program the appropriate saccade to the most informative parts within the object.

Visual information is transmitted along the lateral bank of the IPS from V3A, through LIP, to AIP in the anterior part of the IPS (Nakamura et al., 2001). AIP contains neurons with robust selectivity for disparity-defined, three-dimensional shapes (Srivastava, Orban, and Janssen, unpublished observations). Hence, both $2 \mathrm{D}$ and $3 \mathrm{D}$ shape are processed at end-stage areas of the dorsal and ventral (Janssen et al., 2000) visual stream. The very concept of shape, however, appears to differ in the two processing streams. Whereas the ventral stream computes an abstract, invariant representation of shape for object recognition and categorization, the dorsal stream may represent shape in a more action-oriented manner (Goodale and Westwood, 2004): the representation of shape seems intimately linked to the representation of visual information (position and size) critical for the 
(potential) ensuing action. This would at least partially explain the joint (and admittedly confusing) representation of shape and position we observed in this study. Future studies will have to elucidate the basic nature of the shape representation in the dorsal visual stream.

\section{References}

Andersen RA, Buneo CA (2002) Intentional maps in posterior parietal cortex. Annu Rev Neurosci 25:189-220.

Andersen RA, Asanuma C, Essick G, Siegel RM (1990) Corticocortical connections of anatomically and physiologically defined subdivisions within the inferior parietal lobule. J Comp Neurol 296:65-113.

Avillac M, Denève S, Olivier E, Pouget A, Duhamel JR (2005) Reference frames for representing visual and tactile locations in parietal cortex. Nat Neurosci 8:941-949.

Barash S, Bracewell RM, Fogassi L, Gnadt JW, Andersen RA (1991) Saccaderelated activity in the lateral intraparietal area. II. Spatial properties. J Neurophysiol 66:1109-1124.

Ben Hamed S, Duhamel JR, Bremmer F, Graf W (2001) Representation of the visual field in the lateral intraparietal area of macaque monkeys: a quantitative receptive field analysis. Exp Brain Res 140:127-144.

Bisley JW, Krishna BS, Goldberg ME (2004) A rapid and precise onresponse in posterior parietal cortex. J Neurosci 24:1833-1838.

Borra E, Belmalih A, Calzavara R, Gerbella M, Murata A, Rozzi S, Luppino G (2008) Cortical connections of the macaque anterior intraparietal (AIP) area. Cereb Cortex 18:1094-1111.

Brincat SL, Connor CE (2004) Underlying principles of visual shape selectivity in posterior inferotemporal cortex. Nat Neurosci 7:880-886.

Deneve S, Latham PE, Pouget A (2001) Efficient computation and cue integration with noisy population codes. Nat Neurosci 4:826-831.

Durand JB, Nelissen K, Joly O, Wardak C, Todd JT, Norman JF, Janssen P, Vanduffel W, Orban GA (2007) Anterior regions of monkey parietal cortex process visual shape. Neuron 55:493-505.

Goodale MA, Milner AD (1992) Separate visual pathways for perception and action. Trends Neurosci 15:20-25.

Goodale MA, Westwood DA (2004) An evolving view of duplex vision: separate but interacting cortical pathways for perception and action. Curr Opin Neurobiol 14:203-211.

Goodale MA, Milner AD, Jakobson LS, Carey DP (1991) A neurological dissociation between perceiving objects and grasping them. Nature 349:154-156.

Goodwin AW, Jenmalm P, Johansson RS (1998) Control of grip force when tilting objects: effect of curvature of grasped surfaces and applied tangential torque. J Neurosci 18:10724-10734.

Gottlieb JP, Kusunoki M, Goldberg ME (1998) The representation of visual salience in monkey parietal cortex. Nature 391:481-484.

Hikosaka O, Wurtz RH (1983) Visual and oculomotor functions of monkey substantia nigra pars reticulata. III. Memory-contingent visual and saccade responses. J Neurophysiol 49:1268-1284.

Ito M, Tamura H, Fujita I, Tanaka K (1995) Size and position invariance of neuronal responses in monkey inferotemporal cortex. J Neurophysiol 73:218-226.

James TW, Culham J, Humphrey GK, Milner AD, Goodale MA (2003) Ventral occipital lesions impair object recognition but not object-directed grasping: an fMRI study. Brain 126:2463-2475.

Janssen P, Shadlen MN (2005) A representation of the hazard rate of elapsed time in macaque area LIP. Nat Neurosci 8:234-241.

Janssen P, Vogels R, Orban GA (2000) Three-dimensional shape coding in inferior temporal cortex. Neuron 27:385-397.

Jenmalm P, Johansson RS (1997) Visual and somatosensory information about object shape control manipulative fingertip forces. J Neurosi 17:4486-4499.

Kovacs G, Vogels R, Orban GA (1995) Selectivity of macaque inferior temporal neurons for partially occluded shapes. J Neurosci 15:1984-1997.

Lagae L, Maes H, Raiguel S, Xiao DK, Orban GA (1994) Responses of macaque STS neurons to optic flow components: a comparison of areas MT and MST. J Neurophysiol 71:1597-1626.

Lehky SR, Sereno AB (2007) Comparison of shape encoding in primate dorsal and ventral visual pathways. J Neurophysiol 97:307-319.

Leon MI, Shadlen MN (2003) Representation of time by neurons in the posterior parietal cortex of the macaque. Neuron 38:317-327.

Lewis JW, Van Essen DC (2000) Mapping of architectonic subdivisions in the macaque monkey, with emphasis on parieto-occipital cortex. J Comp Neurol 428:79-111.

Logothetis NK, Sheinberg DL (1996) Visual object recognition. Annu Rev Neurosci 19:577-621.

Maunsell JH, Gibson JR (1992) Visual response latencies in striate cortex of the macaque monkey. J Neurophysiol 68:1332-1344.

Murata A, Gallese V, Luppino G, Kaseda M, Sakata H (2000) Selectivity for the shape, size, and orientation of objects for grasping in neurons of monkey parietal area AIP. J Neurophysiol 83:2580-2601.

Mysore SG, Vogels R, Raiguel SE, Orban GA (2006) Processing of kinetic boundaries in macaque V4. J Neurophysiol 95:1864-1880.

Nakamura H, Kuroda T, Wakita M, Kusunoki M, Kato A., Mikami A., Sakata H., Itoh K (2001) From three-dimensional space vision to prehensile hand movements: the lateral intraparietal area links the area V3A and the anterior intraparietal area in macaques. J Neurosci 21:8174-8187.

Op De Beeck H, Vogels R (2000) Spatial sensitivity of macaque inferior temporal neurons. J Comp Neurol 426:505-518.

Pare M, Wurtz RH (1997) Monkey posterior parietal cortex neurons antidromically activated from superior colliculus. J Neurophysiol 78:34933497.

Platt ML, Glimcher PW (1999) Neural correlates of decision variables in parietal cortex. Nature 400:233-238.

Rainer G, Asaad WF, Miller EK (1998) Selective representation of relevant information by neurons in the primate prefrontal cortex. Nature 393:577-579.

Sakata H, Taira M, Murata A, Mine S (1995) Neural mechanisms of visual guidance of hand action in the parietal cortex of the monkey. Cereb Cortex 5:429-438.

Sáry G, Vogels R, Orban GA (1993) Cue-invariant shape selectivity of macaque inferior temporal neurons. Science 260:995-997.

Schwartz EL, Desimone R, Albright TD, Gross CG (1983) Shape recognition and inferior temporal neurons. Proc Natl Acad Sci USA 80:5776-5778.

Sereno AB, Amador SC (2006) Attention and memory-related responses neurons in the lateral intraparietal area during spatial and shape-delayed match-to-sample tasks. J Neurophysiol 95:1078-1098.

Sereno AB, Maunsell JHR (1998) Shape selectivity in primate lateral intraparietal cortex. Nature 395:500-503.

Shadlen MN, Newsome WT (1996) Motion perception: seeing and deciding. Proc Natl Acad Sci USA 93:628-633.

Shadlen MN, Newsome WT (2001) Neural basis of a perceptual decision in the parietal cortex (area LIP) of the rhesus monkey. J Neurophysiol 86:1916-1936.

Snyder LH, Batista AP, Andersen RA (1997) Coding of intention in the posterior parietal cortex. Nature 386:167-170.

Tanaka K (1996) Inferotemporal cortex and object vision. Annu Rev Neurosci 19:109-139.

Ungerleider LG, Mishkin M (1982) Two cortical visual systems. In: Analysis of visual behavior (Ingle DJ, Goodale MA, Mansfield RJW, eds), pp 549586. Cambridge, MA: MIT. 